\title{
Correction to: How Resiliency and Hope Can Predict Stress of Covid-19 by Mediating Role of Spiritual Well-being Based on Machine Learning
}

\author{
Roghieh Nooripour $^{1}$ (D) Simin Hosseinian ${ }^{1} \cdot$ Abir Jaafar Hussain $^{2}$. \\ Mohsen Annabestani ${ }^{3}$. Ameer Maadal ${ }^{4}$. Laurel Radwin ${ }^{5}$. \\ Peyman Hassani-Abharian ${ }^{6} \cdot$ Nikzad Ghanbari $^{7} \cdot$ Abolghasem Khoshkonesh $^{8}$ \\ Published online: 19 January 2021 \\ (c) Springer Science+Business Media, LLC, part of Springer Nature 2021
}

\section{Correction to: Journal of Religion and Health (2020) https://doi.org/10.1007/s10943-020-01151-z}

The original version of the article was inadvertently published with the ethical code in Ethical Considerations section. This has been corrected with this erratum. The revised texts are given below:

\section{Ethical Considerations}

The present study was carried out following the approval of the Ethics Committee at Alzahra University, Iran, in 2020. All of the procedures conducted in this research with humans is consistent with the National Research Committee's ethical standards, the Helsinki Declaration of 1964 and its subsequent revisions, or equivalent ethical norms. Informed consent and participants' information sheet indicated the right for the participants to withdraw at any point of the conducted research.

The original article has been corrected.

Publisher's Note Springer Nature remains neutral with regard to jurisdictional claims in published maps and institutional affiliations.

The original article can be found online at https://doi.org/10.1007/s10943-020-01151-z.

Roghieh Nooripour

r.noori@alzahra.ac.ir

Extended author information available on the last page of the article 


\section{Affiliations}

\section{Roghieh Nooripour $^{1}$ (D) Simin Hosseinian ${ }^{1} \cdot$ Abir Jaafar Hussain $^{2}$. Mohsen Annabestani ${ }^{3}$. Ameer Maadal ${ }^{4}$. Laurel Radwin ${ }^{5}$. Peyman Hassani-Abharian $^{6} \cdot$ Nikzad Ghanbari $^{7} \cdot$ Abolghasem Khoshkonesh $^{8}$}

Simin Hosseinian hosseinian@alzahra.ac.ir

Abir Jaafar Hussain

a.hussain@ljmu.ac.uk

Mohsen Annabestani annabestany2@gmail.com

Ameer Maadal amir.moaddel135@gmail.com

Laurel Radwin

laurel.e.radwin3@gmail.com

Peyman Hassani-Abharian abharian1972.hasan@yahoo.com

Nikzad Ghanbari

nik.ghanbari@gmail.com

Abolghasem Khoshkonesh

khoshkonesh.abolghasem@yahoo.com

1 Department of Counseling, Faculty of Education and Psychology, Alzahra University, Tehran, Iran

2 Computer Science Department, Liverpool John Moores University, Liverpool L33AF, UK

3 Faculty of Electrical Engineering, Sharif University of Technology, Azadi Ave, PO Box 11365-11155, Tehran, Iran

4 Department of Psychology, Faculty of Humanities, Islamic Azad University, Shahr-E Rey Branch, Tehran, Iran

5 Center for Health Care Organizational and Implementation Research (CHOIR), VA Boston Healthcare System, Boston, MA, USA

6 Institute for Cognitive Science Studies, Brain and Cognition Clinic, Tehran, Iran

7 Clinical Psychology (Prevention and Treatment of Addiction), Faculty of Education and Psychology, Shahid Beheshti University (SBU), Tehran, Iran

8 Department of Counseling, Faculty of Education and Psychology, Shahid Beheshti University (SBU), Tehran, Iran 